\title{
Culture et élevage : quelles relations, quelles synergies ?
}

Oléagineux, Corps Gras, Lipides. Volume 11, Numéro 4, 261-7, JUILLET-OCTOBRE 2004, AGRICULTURE / ÉLEVAGE : NORD-SUD

\section{Auteur(s) : Olivier LAPIERRE*}

Département des Sciences animales de I'INA P-G, Centre d'Etude et de Recherche sur l'Economie et I'Organisation des Productions Animales (CEREOPA), 16, rue Claude Bernard F75231 Paris Cedex 05

Résumé : AbstractEven if they seem natural, the relationships between crop productions and livestock productions are not yet very developed. The initial conditions of the CAP and environmental constraints did not favour coordinated actions. With some exeptions, the plant and animal fields have no coordinated strategies. The current conditions should support the reinforcement of these relationships. The new economic deal, the new consumption tendancies, and the rise of regional policies encourage them. To use novel scales for novel reasonings and to revise practices of decision are the conditions necessary to this change.

Mots-clés : crop farming, livestock farming, coordination, strategy

\section{ARTICLE}

La question des relations qui existent entre culture et élevage est complexe et délicate à aborder de façon synthétique et exhaustive. Sans y prétendre, le présent article cherche à proposer une lecture de la façon dont elles se sont développées dans le passé et dont elles pourraient évoluer dans l'avenir. II rappelle en quoi le contexte de la PAC d'avant 1992 et les règlements édictés en matière de protection de l'environnement ont contribué à installer une situation où les logiques du végétal et de l'animal se sont, finalement, peu coordonnées. Il explore les perspectives ouvertes par l'apparition de nouveaux enjeux et les conséquences induites en matière de synergies. II traite la question posée de la nécessaire adaptation des modalités de la prise de décision et des outils susceptibles de la servir.Le point de vue de l'auteur est celui d'une personne qui s'est très régulièrement trouvée en situation d'interface entre des opérateurs du monde du végétal et ceux du monde animal. Le caractère prospectif de son propos est nourri de ses propres expériences mais aussi des échanges qu'il a pu avoir, au cours des dernières années, avec des interlocuteurs également préoccupés par ces questions et, comme lui, confiants dans l'opportunité de nouvelles synergies à développer.

\section{Production animale, production végétale : un passé de logiques peu coordonnées}

La situation qui prévaut aujourd'hui est l'héritière d'une évolution qui s'est produite au cours de la seconde moitié du XX $X^{\mathrm{e}}$ siècle [1]. Elle a peu contribué au développement de coordinations entre les logiques mises en œuvre au sein des univers " animal » et " végétal ». A quelques exceptions près, on doit accepter le constat qu'en dépit du fait que l'alimentation animale constitue un débouché 
majeur pour les productions végétales et que les coûts d'alimentation sont l'un des principaux déterminants de la compétitivité des productions animales, les acteurs des deux secteurs ont peu concerté leurs stratégies. C'est le marché qui aura généralement servi de courroie d'entraînement et orienté les choix des uns et des autres.

\section{Un contexte économique et réglementaire très déterminant}

Considérées sur une période longue, les forces qui ont façonné ce jeu de relations ont donc plutôt contribué à le distendre qu'à le renforcer. Les raisons de cette évolution sont nombreuses. Elles relèvent de considérations techniques, économiques et culturelles, qui ont varié dans le temps et dans l'espace, et ne peuvent être développées en détail ici. Certains facteurs ont cependant plus largement contribué à cette dynamique.

\section{La politique agricole commune}

La politique agricole commune (PAC) d'avant 1992 a eu un effet majeur, probablement le plus important, sur cette évolution.

- Le soutien du marché céréalier. Par le biais des mécanismes mis en place pour garantir, sur le marché intérieur, un prix des céréales rémunérateur pour les agriculteurs, le soutien du marché céréalier a eu comme conséquence d'en rendre la consommation par les animaux peu intéressante sur le plan économique. II faut se souvenir qu'avant la réforme de 1992, le prix d'achat du maïs par un utilisateur européen était plus de deux fois plus élevé que celui payé par un utilisateur aux USA.Dans ce contexte, les stratégies d'approvisionnement des filières animales ont consisté à rechercher toutes les alternatives possibles à l'utilisation des céréales. Elles ont abouti au développement des utilisations des PSC (produits de substitution des céréales) en tant que matières premières concentrées utilisées comme sources d'énergie. Elles ont, probablement aussi, abouti à consolider la place du maïs fourrage dans les systèmes alimentaires des élevages de ruminants (laitiers en particulier) où il présentait l'avantage d'un apport énergétique autonome et avantageux sur le plan économique.La conséquence directe de cette situation est que les choix de production et de mise en marché des céréales n'étaient que très peu motivés par la demande des filières animales. Elle est aussi que les filières animales européennes ont su développer une capacité de diversification de leurs ressources alimentaires tout à fait exemplaire. Au début des années 80 , la situation néerlandaise était souvent citée en exemple de cette aptitude. Les filières européennes sont, en cela, très différentes de leurs homologues d'Amérique du Nord. Ces dernières, qui n'ont pas eu d'avantages à valoriser d'autres matières premières que le maïs et le tourteau de soja, sont, en comparaison, toujours restées dans un modèle relativement simple d'approvisionnement.

- Le plan protéine. Lancé en 1978 à la suite de la crise du soja liée à l'embargo américain de 1973, le plan protéine aura, lui aussi, participé de ce jeu de forces contradictoires.Les aides accordées à l'utilisation des protéagineux ou à la déshydratation des fourrages ont largement favorisé l'utilisation de ces matières premières et contribué au maintien d'un prix de marché élevé. Le montant de ces aides étant indexé sur le prix des matières premières concurrentes importées (le soja en particulier), il garantissait un niveau de demande important et relativement stable. Ce contexte de marché a incité les agriculteurs à introduire ces cultures 
dans leurs assolements. Leur prix de marché les rendait intéressantes face aux céréales en termes de marge à l'hectare. Vu sous cet angle, le plan protéine a donc plutôt favorisé les synergies entre système d'élevage et système de culture. Le pois était devenu une ressource majeure pour l'alimentation des porcs, en même temps qu'il offrait aux céréaliers l'avantage d'une tête d'assolement rentable et efficace en matière agronomique.Paradoxalement et dans le même temps, cette politique rendait les fournisseurs peu capables d'apprécier la véritable valeur que leur produit prenait entre les mains de leurs clients. A la fin des années 90, le prix perçu par les producteurs de pois pouvait être plus de deux fois plus élevé que celui "consenti» par les utilisateurs qui bénéficiaient de l'aide apportée par l'Union européenne. A la même période, l'aide accordée à la déshydratation des fourrages permettait au " système luzerne » de réaliser un chiffre d'affaires très supérieur à la dépense supportée par le "système production animale ". A de rares exceptions près, les fournisseurs de l'époque exploitaient une rente sans vraiment prendre la mesure de l'intérêt réel de leur offre de produit.

\section{La politique de protection de l'environnement}

Les mesures prises, depuis les années 70, pour limiter l'impact des activités agricoles sur l'environnement ont également joué leur rôle dans la relation culture-élevage. Ce sont les règles liées à la pratique de l'épandage des effluents par les éleveurs qui ont eu le plus d'effet. Incontestablement, les assolements développés en Bretagne l'ont été en fonction du potentiel d'épuration qu'ils permettaient d'atteindre. La réputation du colza en tant que " pompe à lisier » et le statut du pois, protéagineux non pris en compte dans la surface d'épandage, auront joué un rôle dans la décision des agriculteurs de privilégier telle ou telle rotation. Des simulations montraient assez bien que l'intérêt du pois, dans l'assolement d'une exploitation produisant ses propres ressources alimentaires, diminuait au fur et à mesure que la densité du cheptel augmentait [2]. L'obligation de disposer de surfaces pour épandre ses effluents conduisait à privilégier la production de céréales, même au-delà du besoin des cheptels, et le recours à des achats de soja plutôt qu'à assurer son autonomie protéique en maintenant la production de pois sur les terres de l'exploitation.

\section{Un face à face peu propice au développement de synergies}

Les conditions précédentes ont abouti à ce que se développent, dans chacun des deux univers, des logiques peu favorables aux synergies. C'est le marché qui aura, le plus souvent, servi de courroie de transmission et orienté les choix des uns et des autres vers la situation que l'on connaît actuellement.

\section{Filières animales : la logique de la substituabilité et du nutriment anonyme}

C'est une caractéristique majeure des stratégies d'approvisionnement développées par les filières animales européennes que d'avoir exacerbé le principe de substituabilité entre les matières premières (encadré 1). Dans un contexte d'approvisionnement difficile, la compétitivité des entreprises résidait principalement dans leur capacité à savoir tirer parti de toutes les opportunités qui se présentaient sur le marché. Tablant sur le principe du " nutriment anonyme " qui revient à considérer que les nutriments fournis par les différentes matières premières sont équivalents («l'énergie du blé vaut celle du manioc »), et en adoptant des pratiques de révision fréquente des formules alimentaires par le moyen des techniques d'optimisation, ils ont mis en oeuvre des 
politiques d'approvisionnement où la place relative des différentes matières premières était susceptible de varier très rapidement et de façon très importante.

Les compétences clefs d'une telle politique étaient :

- - l'achat, qui devait garantir, à tout moment, le meilleur prix ;

- - la formulation, qui devait recalculer, périodiquement, la combinaison de matières premières la plus avantageuse ;

- - l'évaluation des matières premières qui devait permettre, très rapidement, d'apprécier l'intérêt zootechnique de tout nouveau produit présenté sur le marché.

En conséquence, les acteurs des filières animales étaient peu enclins à s'engager, sur le long terme, à l'égard de leurs fournisseurs. Leur comportement était majoritairement de type " pick » (encadré 2). Ils s'abritaient même volontiers derrière une politique du secret qui ne les incitait pas à expliciter leurs attentes. Le marketing amont en était souvent réduit à sa plus simple expression : fixer un prix et des conditions de livraison. Cette politique d'achat se différenciait de celle de leurs collègues des entreprises de l'alimentaire, les meuniers par exemple. Ces derniers, incapables de jouer la substituabilité entre matières premières, privilégiaient des politiques d'achat plus stables et faisaient la part belle à la qualité. Cette différence de comportement a contribué à imposer une image parfois négative du secteur de l'alimentation animale dans l'esprit de ses fournisseurs.

\section{Filières végétales : la logique de la livraison à un marché mal compris}

Face aux acheteurs des filières animales, les fournisseurs des filières végétales n'ont pas non plus pris la mesure du marché sur lequel ils trouvaient pourtant une part importante de leurs débouchés. Face à des clients dont le comportement d'achat apparaissait comme très fluctuant et parfois même incompréhensible, sûrs d'avoir des débouchés par des mécanismes tels que l'intervention et peu à même d'apprécier la véritable valeur de leur produit pour leurs clients, les acteurs des filières végétales n'ont que rarement engagé des efforts pour adapter la nature de leur offre aux attentes des filières animales.

Cette relative incompréhension était particulièrement sensible en matière de politique de qualité et de fonctionnement du marché.

Les filières végétales ont eu beaucoup de difficultés à percevoir que la valeur énergétique des matières premières et leur densité nutritionnelle étaient les premières qualités recherchées par les utilisateurs [3]. Dans un contexte où la question de l'autonomie protéique était souvent mise sur le devant de la scène, il leur semblait paradoxal de devoir faire porter des efforts d'amélioration d'abord sur la valeur énergétique de leur produit. Cette incompréhension a pu se manifester à propos des questions d'amélioration génétique. La luzerne et le maïs en sont des exemples. Le rendement en matière à l'hectare s'est plus souvent imposé comme critère de sélection que la densité énergétique recherchée par les utilisateurs. Elle s'est aussi manifestée à travers le fait que les politiques de segmentation de l'offre à destination de l'alimentation animale sont restées très marginales dans l'univers des organismes de collecte. 
Face au fonctionnement des marchés, les fournisseurs de matières premières restaient souvent perplexes. Les triturateurs, fournisseurs de tourteaux mais consommateurs de graines, avaient des difficultés à comprendre les raisons qui amenaient les fabricants d'aliments du bétail à proposer, à certaines périodes (fin des années 90 ), des prix aussi élevés pour les graines. Les vendeurs de pois protéagineux se sont montrés difficiles à convaincre que le prix de leur produit était principalement déterminé par celui du tourteau de soja alors que l'observation, sur période longue, de sa dynamique de prix prouvait qu'il était d’abord influencé par celui du blé [4].

\section{Des contre-exemples édifiants}

La réalité des faits ne peut cependant pas se limiter aux explications précédentes. Au sein du système agricole, et au-delà du cas des éleveurs engagés dans la production de leurs propres ressources alimentaires, des contre-exemples intéressants ont existé. Ils sont probablement, pour certains d'entre eux, les précurseurs de solutions qui pourraient se développer dans le futur.

- Du côté des fournisseurs de matières premières, la luzerne et le corn-gluten-feed sont des preuves très convaincantes de l'intérêt du développement d'une politique de qualité spécifiquement destinée au secteur de l'alimentation animale. Initiées par deux entreprises, France Luzerne et Roquettes, ces politiques ont abouti à l'apparition sur le marché de grades de qualité, pour la luzerne, ou de spécifications techniques précises, pour le CGF. Dans un cas comme dans l'autre, c'est le choix des entreprises d'aller directement sur le marché, plutôt que de concéder la vente à des négociants ou de passer par des courtiers, qui aura favorisé leur capacité à mieux percevoir ses attentes. A l'époque, la faiblesse des équipes commerciales dédiées au secteur de l'alimentation animale, était plutôt la règle du côté des entreprises de l'univers végétal.

- Du côté des utilisateurs de matières premières, la valorisation, par les fabricants d'aliment du bétail, des céréales produites sur les exploitations agricoles des éleveurs constitue un autre exemple. Initialement motivé par l'objectif d'économie des taxes imposées lors de la vente des marchandises, l'échange céréales/aliment s'est maintenu après la réforme de 1992, même si son intérêt économique immédiat devenait moins important. II traduisait la volonté des industriels de l'alimentation animale d'adhérer à la logique des éleveurs, producteurs de céréales, soucieux de mettre en place des rotations plus susceptibles d'assurer leur autonomie alimentaire. Cette coordination s'est même renforcée par la mise en place de contrats qui assuraient aux producteurs de céréales livrant leur production aux fabricants d'aliments du bétail des niveaux de rémunération plus intéressants en contrepartie du respect, par eux, d'un certain nombre de règles de production visant à améliorer la qualité de leur produit.

- L'échelle de la mise en œuvre de ces initiatives s'est le plus souvent cantonnée au niveau de l'entreprise et de ses relations commerciales directes. En dehors de quelques exemples de productions animales, conduites dans un contexte d'AOC, les échelles de la région ou de la filière n'ont pratiquement jamais été concernées. 


\section{Nouveaux enjeux, nouvelles synergies}

Si la situation actuelle, fruit des évolutions passées, est plutôt caractérisée par la coexistence de logiques peu coordonnées, elle n'en est pas pour autant figée. Au contraire, des signes évidents d'évolution sont perceptibles. Des initiatives sont en phase de développement ou sur le point de naître qui permettent d'envisager que de nouvelles synergies se créent entre les univers du végétal et de l'animal.

\section{Nouveau contexte, nouvelles préoccupations}

Les perturbations majeures provoquées par les crises alimentaires, la réforme de la PAC et de l'OMC, qui sont survenues au cours des dernières années dans l'environnement des systèmes de production agricole ont modifié les perspectives des acteurs et fait émerger tout un ensemble de préoccupations nouvelles. Face à une nouvelle donne économique, en réponse aux attentes de consommateurs de plus en plus exigeants et sous l'impulsion de dynamiques régionales de plus en plus affirmées, on voit se définir un nouveau contexte dans lequel la coordination entre les logiques du végétal et de l'animal pourrait se renforcer.

\section{Une nouvelle donne économique}

La réforme de la PAC de 1992 a profondément transformé les conditions d'approvisionnement en matières premières des filières animales. La baisse programmée du prix des céréales n'a pas simplement contribué à restaurer leur compétitivité sur le marché de l'alimentation animale [5]. Elle a aussi eu pour conséquence de modifier l'état d'esprit dans lequel les acteurs de ces deux secteurs se considèrent mutuellement.

Pour les producteurs de matières premières, l'intérêt relatif de la valorisation de leur production via l'alimentation animale les amène à considérer ce débouché avec plus d'attention que par le passé. Sans être pour autant enclins, à de rares exceptions près, à s'engager dans la politique de valorisation directe à laquelle ils avaient initialement pu penser, ils mettent en œuvre des actions qui tendent à renforcer leur position sur ce marché. C'est particulièrement le cas dans le domaine de la qualité sanitaire et, dans une moindre mesure, dans celui de la qualité nutritionnelle pour laquelle certains d'entre eux ont engagé des démarches de différenciation.

Du côté des utilisateurs de matières premières, la baisse du prix des céréales a eu au moins deux conséquences. Elle s'est d'abord traduite par un développement significatif des utilisations. Les pourcentages d'incorporation dans certaines formules ont atteint de tels niveaux que la question de la qualité et donc des conditions de production et de stockage se pose avec une acuité nouvelle. La seconde conséquence est que, dans ce nouveau contexte de prix, les avantages de leur substitution par des produits plus intéressants sur le plan économique se sont singulièrement réduits. Même s'il peut encore, à certaines périodes, s'avérer intéressant d'abandonner l'utilisation du blé ou du maïs, l'économie réalisée est maintenant beaucoup plus faible que par le passé. Ainsi, le surcoût d'une politique d'approvisionnement plus structurelle, qui limiterait les possibilités de substitution d'une matière première par une autre, devient plus facilement acceptable. 


\section{Répondre aux attentes des consommateurs/citoyens}

Les difficultés rencontrées au cours des dernières années incitent les filières animales à mieux tenir compte de la façon dont évolue la demande des consommateurs. Au-delà du constat des changements, elles cherchent aujourd'hui à adapter leurs modes de production pour être plus en adéquation avec ces nouvelles attentes. Sans négliger l'importance du prix, qui reste pour le consommateur le premier déterminant de ses choix, on peut évoquer quatre autres enjeux majeurs. Leur prise en compte par les filières animales pourrait les amener à développer de nouvelles formes de relations avec leurs fournisseurs de matières premières.

- Sécurité sanitaire. L'enjeu de la sécurité sanitaire est probablement celui qui a pris le plus d'importance au cours de la dernière décennie. Sa prise en compte, quand elle n'est pas seulement vécue uniquement comme une question de transfert de responsabilités, peut conduire à développer de nouvelles relations entre le maillon de l'élevage et celui de la production végétale. Compte tenu des exigences de traçabilité revendiquées par les consommateurs et les distributeurs, il existe de véritables opportunités d'innovation. L'engagement d'un distributeur tel que Carrefour dans la mise en place d'une filière tracée pour l'approvisionnement en tourteaux de soja en est un exemple emblématique. En dehors de la question des OGM qui continue de focaliser les attentions, il existe d'autres opportunités. Le fait que des opérateurs situés très en amont de la chaîne (fournisseurs de phytosanitaires ou sélectionneurs végétaux) aient développé des démarches de filière est assez révélateur d'un changement qui devrait permettre aux opérateurs du système agricole de reprendre l'initiative dans un domaine qui reste aujourd'hui surtout exploité par la grande distribution. La création récente de RESEDA (association professionnelle pour conforter la qualité et la sécurité des denrées animales) préfigure aussi cette évolution. Le fait d'y retrouver, en plus des trois membres fondateurs que sont I'ANIA, le SNIA et le SYNCOPAC, deux organisations interprofessionnelles, le CNIEL (filière lait) et INTERBEV (filière viande), sept organisations professionnelles: I'AFCA-CIAL pour les aliments minéraux, les prémix et autres compléments de la nutrition animale, le SIMV pour les médicaments vétérinaires, I'UIPP pour les produits de protection des plantes, le SDVF pour la vitellerie française, le SIFCO pour les coproduits animaux, VALORIA pour la valorisation des produits dérivés de I'industrie alimentaire, la FNADE pour les activités de dépollution et de l'environnement et un institut technique, ARVALIS Institut du végétal, est bien la preuve d'un changement de logique des acteurs.

- Santé. Outre son innocuité, le consommateur attend de son alimentation qu'elle contribue positivement à sa santé. Le développement, depuis quelques années, des alicaments, nutraceutiques et autres régimes crétois illustre une tendance qui peut maintenant être perçue comme lourde et durable. Dans cette perspective de l'alimentation santé, les produits animaux ne sont pas les mieux lotis. Leurs matières grasses, en particulier, les placent souvent au banc des accusés. Par l'alimentation des animaux, il est possible de remédier, au moins en partie, à ces inconvénients. Les profils matières grasses des produits animaux (viande, lait ou œuf) sont modulables par l'alimentation (encadré 3 ). Ces possibilités d'effet santé de l'alimentation du bétail sont, dès à présent, mises en œuvre dans plusieurs domaines.Le groupe Glon Sanders vient d'annoncer la mise en marché d'un œuf revendiquant plusieurs avantages nutritionnels. Le mode d'alimentation des pondeuses est à 
la base de ce développement.Depuis plusieurs années, l'entreprise Valorex propose de valoriser, en avantages nutritionnels pour le consommateur, les conséquences sur le profil en acides gras des produits animaux de l'incorporation de graines de lin dans leur alimentation. Le développement d'une marque commerciale a accompagné cette stratégie où sont associés des opérateurs du végétal et de l'animal.

- Terroir. La référence à un terroir est une autre attente forte des consommateurs. Même quand elle est traduite en signe officiel de qualité, elle n'est pas systématiquement accompagnée d'une pratique d'alimentation elle aussi régionale. Or, on voit aujourd'hui des acteurs de ces filières animales régionales explorer l'intérêt de privilégier des ressources alimentaires locales. Les initiatives de la filière des volailles fermières de Loué (grain de terroir) et les projets de l'interprofession du porc en Aquitaine sont deux exemples de la façon dont cette dimension terroir peut modifier la stratégie d'approvisionnement des filières animales. Au-delà des avantages que cela peut leur apporter en matière de traçabilité, c'est aussi pour eux une façon de conforter un positionnement face à des consommateurs attentifs à cette valeur terroir.Facile à développer pour la partie des matières premières énergétiques, cette volonté d'approvisionnement local est plus délicate à satisfaire pour ce qui concerne la fraction protéique de la ration [6]. Le remplacement du tourteau de soja qu'elle implique peut conduire à promouvoir localement des cultures peu développées ou à organiser différemment la valorisation de cultures déjà implantées.La politique de valorisation du colza, produit dans la zone de l'AOC Epoisse, est un exemple de l'effort de coordination qu'impose une telle stratégie. Les graines récoltées dans la zone sont triturées de façon différenciée afin que le tourteau, résultant de l'opération, puisse être utilisé dans l'élaboration des rations en conformité avec le cahier des charges de l'appellation.Le modèle féverole-colza, testé en 2003 sur la ferme de Grignon, constitue un autre exemple de la façon d'adapter la relation entre production végétale et production animale. II intéresse les acteurs de la filière Brie du sud-est de l'lle-de-France dans la mesure où il leur permettrait de mettre en place une solution d'alimentation fondée sur des matières premières régionales et qui serait plus conforme à l'image qu'ils veulent donner de leur produit.

- Ethique. Dernière tendance de consommation à se développer, la consommation éthique peut aussi inciter des acteurs des filières animales à reconsidérer leurs pratiques d'approvisionnement en matières premières. Deux aspects de cette dimension éthique peuvent le justifier.L'environnement est la première. Au-delà même du cadre réglementaire qui s'est durci au cours des dernières années et continuera de le faire, l'affichage de pratiques respectueuses de l'environnement devient un moyen de différenciation de plus en plus fréquent. La mise en œuvre de ces pratiques ne concerne pas seulement l'exploitation des animaux. Pour les filières animales, elle pourrait remonter, en amont, aux conditions de production (itinéraires techniques) des matières premières végétales introduites dans les aliments.L'équité est la seconde. La revendication d'une garantie de rémunération pour les acteurs, les agriculteurs, impliqués dans la filière de production s'est développée sous la forme du commerce équitable, aujourd'hui limité à des productions du sud (café, thé, bananes, etc.). L'élargissement de ce concept à des filières de production moins exotiques 
n'est pas à exclure. Garantir le revenu des producteurs, éleveurs mais aussi producteurs végétaux, associés dans une filière de production, pourra, demain, participer d'une stratégie de positionnement permettant de se différencier sur le marché.

\section{Le développement régional}

L'importance croissante prise par les régions dans la définition des politiques de développement est un autre élément qui peut conduire au renforcement des liens entre élevage et culture.

La volonté d'associer, localement, des activités qui maximisent le potentiel de création de valeur est souvent inscrite dans les projets de développement régional. Il est évident que de telles politiques ne peuvent que favoriser des initiatives susceptibles de valoriser, dans l'alimentation des cheptels régionaux, des ressources alimentaires élaborées localement.

Les politiques d'aménagement du territoire et leur volet environnemental sont un autre axe d'intervention qui peut aussi contribuer au renforcement de ces relations. La définition locale des pratiques éligibles au titre des mesures agro-environnementales en est un exemple. En favorisant le développement de telle ou telle culture (protéagineux par exemple), elle peut favoriser le développement des synergies locales entre culture et élevage.

\section{Tout reste à faire}

Si les conditions actuelles semblent incontestablement plus favorables à l'émergence d'un nouveau système de relations entre les activités d'élevage et celles de production végétale, et si des exemples concrets apportent la preuve de la faisabilité et de l'intérêt de ces changements, il semble quand même qu'elles impliquent des révisions importantes des logiques d'acteurs.

\section{Changement d'échelle des coordinations}

Un premier changement concerne l'échelle à laquelle ces synergies sont mise en œuvre. Par rapport à la situation passée, il apparaît évident qu'on verra se renforcer des coordinations, de filière d'une part, régionales d'autre part.

Les coordinations de filière sont celles qui devraient pouvoir se mettre en place le plus facilement. Justifiées et motivées par un objectif précis de positionnement sur un marché, elles peuvent espérer y récupérer les ressources financières nécessaires à leur mise en œuvre. L'apparition de nouvelles attentes des consommateurs offre, en ce domaine, des opportunités immédiates.

Les coordinations de territoire auront probablement plus de difficultés à se développer. Elles ne bénéficieront pas toujours de l'effet d'entraînement que représente l'attraction du marché. Elles risquent aussi d'être confrontées à la question de l'absence de pilote. Par contre, elles profiteront des aptitudes naturelles à la concertation qui naissent de la proximité. La féverole est plus facile à introduire dans une formule alimentaire par un fabricant d'aliment du bétail, quand une politique locale en encourage la culture et conduit à une amélioration des conditions économiques et techniques de sa collecte.

Ces nouvelles formes d'organisation sont encouragées par le souhait des acteurs des filières de reprendre en partie l'initiative face à la grande distribution. Il est en effet surprenant de constater que ce sont plutôt les enseignes de la grande distribution qui ont eu, dans ce domaine, le courage de 
l'innovation. L'exemple de la mise en place d'une filière soja non OGM par l'entreprise Carrefour est tout à fait révélateur.

\section{Du statut de commodité à celui de spécialité, du marché au contrat : vers une démarche d'ingénierie reverse}

Ces changements ne posent pas uniquement des questions d'organisation. Ils impliquent aussi des changements d'attitude qui vont à l'encontre des habitudes développées au sein des entreprises. Dans une certaine mesure, c'est à un changement de " recette stratégique » qu'ils appellent. C'est la politique d'approvisionnement des filières animales qui devrait connaître les changements les plus radicaux (encadré 2 ). Dans I'hypothèse où elle ne consisterait plus à s'adresser à un marché de commodités peu spécifiées mais à contracter, à moyen ou long terme, pour la fourniture des spécialités nécessaires au positionnement choisi par l'entreprise, elle nécessitera le développement de nouvelles compétences. Elle réclamera aux acteurs de l'élevage de s'impliquer auprès des cultivateurs pour discuter, avec eux, de leurs options techniques. Elle les incitera à développer des systèmes de rémunération moins volatils que le marché et seuls susceptibles de leur garantir la régularité quantitative et qualitative de leurs approvisionnements.

Mais, pour avoir des chances d'aboutir, cette dynamique devra abandonner le terrain d'une compétitivité principalement fondée sur le prix. Sans avoir la naïveté de penser que ce dernier n'aura plus d'importance, il faut oser imaginer que pourront se développer des relations entre acteurs dont il ne sera pas le seul arbitre. Cela devra être le cas du côté des consommateurs. Cela devra aussi être le cas plus en amont des filières, où les opérateurs devront accepter l'idée que la rentabilité de leur activité ne dépende pas de leur seul pouvoir de négociation, au coup par coup, sur un marché volatil. Vu l'état actuel du fonctionnement des marchés des matières premières de l'alimentation animale, le pari n'est pas gagné.

\section{Nouvelles pratiques de décision, nouvelles démarches d'expérimentation}

La révision des outils d'aide à la décision devra immanquablement accompagner la mise en place de ces nouvelles politiques d'approvisionnement. Les démarches d'optimisation actuellement mises en œuvre par les entreprises d'aliment du bétail peuvent aussi servir à l'arbitrage au sein de situations plus complexes dans lesquelles sont associées des questions de choix de production végétale et des questions de formulation de mélanges alimentaires (encadré 4). D'autres approches sont également susceptibles d'être mobilisées [7]. Elles doivent favoriser la prise en compte simultanée de points de vue complémentaires mais différents. Dans un environnement de "logiques transversales", elles doivent permettre la mise en œuvre d'une véritable " dialectique projective ", en tant que processus d'équilibration et de résolution des conflits d'intérêt [8]. L'arsenal des outils de traitement de l'information (dynamique des systèmes, systèmes multi-agents, etc.) est riche de techniques qui pourront être déployées au service de la conception et de la réalisation de nouveaux outils d'aide à la décision. Les questions de gouvernance associées à la problématique du développement durable pourraient trouver, dans ce domaine, un sens très opérationnel.

Mais, ce ne sont pas seulement les règles de décision qui doivent être adaptées à ces nouvelles donnes. En amont les systèmes de production des connaissances et des référentiels technicoéconomiques doivent aussi les prendre en compte. Or, force est de constater que ces systèmes sont aujourd'hui organisés sur des bases qui ressemblent fort à celles des acteurs économiques. Leurs 
logiques sont encore peu coordonnées. Que ce soit au niveau de la recherche appliquée, du développement ou de la démonstration, il devient indispensable de mettre en place des programmes et des initiatives qui permettent d'avoir une démarche plus intégrée. Le programme mis en place sur la ferme expérimentale de l'INA P-G à Grignon est une illustration, dans le domaine de la production laitière, de ce qui devrait se généraliser. Du champ à l'assiette, en passant par les maillons de l'étable et de l'usine, il propose une approche globale et intégrée, pour traiter de questions de systèmes de culture, de stratégie d'alimentation et de développement de produits laitiers mieux positionnés en matière de valeur santé. La généralisation de telles approches nécessitera plus de collaboration entre les stations de recherche ou entre les centres techniques. Elle pourrait aussi justifier que les infrastructures soient remaniées pour offrir des possibilités de prise en compte simultanée des différentes dimensions de cette globalité.

\section{Conclusion}

L'explication du manque de synergies, au sein de notre système de production agricole, entre les productions animales et les productions végétales est facile à donner. Le contexte économique et réglementaire dans lequel se sont développées ces activités ne les a pas encouragées, bien au contraire. Même si, çà et là, des initiatives ont pu montrer l'intérêt de les rechercher et la faisabilité de leur développement, nous sommes aujourd'hui dans une situation où les logiques des acteurs sont assez peu coordonnées.

Cette situation devrait pourtant évoluer. Les mutations survenues dans l'environnement de nos activités de production sont telles qu'ils encouragent au changement. Pour de multiples raisons, on perçoit bien les avantages qui pourraient naître d'une meilleure coordination des stratégies de développement des acteurs. II nous semble qu'il est temps de sortir d'un modèle où chacun met au point, dans son coin, sa propre stratégie et va chercher, sur le marché, la validation de ses choix, pour rentrer dans un modèle où, en synergie avec des partenaires d'amont et d'aval, il entreprendra la coconstruction de solutions répondant mieux aux nouveaux enjeux et plus profitables. C'est peutêtre dans ce changement de recette stratégique que les productions agricoles européennes et françaises trouveront, demain, les moyens de relever le défi d'une compétition accrue.

\section{Références}

1 COLEOU J. L'apparition des modes intensifs d'élevage. In : Le mangeur et l'animal Mutations de l'élevage et de la consommation. Paris : Autrement, Coll. Mutations/Mangeurs, 1997 : 42-68; n 172.

2 LAPIERRE O. Stratégies d'approvisionnement en matières premières des filières animales : nouveaux jeux de contraintes, nouvelles pratiques de décision. In : CR colloque annuel Valicentre (18 novembre 1999). 1999 : 75-9.

3 DELPLANCKE D, LAPIERRE O. L'approvisionnement européen en protéines : un handicap relatif ? OCL $1998 ; 5\left(n^{\circ} 4\right): 255-61$.

4 LAPIERRE O, HUARD M. Déterminants de la consommation des matières riches en protéines en alimentation animale. OCL $1996 ; 3\left(n^{\circ} 5\right): 327-31$. 
5 DRONNE Y. Avenir du secteur de l'alimentation animale en relation avec les filières végétales. INRA Prod Anim $2003 ; 16(5)$ : 333-40.

6 LAPIERRE O, PRESSENDA F. Adaptation des stratégies d'approvisionnement des filières animales en matières premières riches en protéines. OCL $2002 ; 9\left(n^{\circ} 2-3\right): 86-91$.

7 CLOUTIER LM, SONKA ST. Dynamique de système et gestion stratégique d'une chaîne de valeur ajoutée agro-alimentaire. In : La dynamique des systèmes. Paris : Hermès, 1998 : 43-56.

8 MELESE J. Approches systémiques des organisations, vers l'entreprise à complexité humaine. Paris : Les éditions d'organisation (Collection Les classiques EO), 1979 ; 1990. 